\title{
Detection of Rotor Forced Response Vibrations Using Stationary Pressure Transducers in a Multistage Axial Compressor
}

\author{
William L. Murray III and Nicole L. Key \\ Purdue University, 500 Allison Road, West Lafayette, IN 47907, USA \\ Correspondence should be addressed to Nicole L. Key; nkey@purdue.edu
}

Received 23 July 2014; Revised 31 October 2014; Accepted 3 November 2014

Academic Editor: Mark McQuilling

Copyright ( 2015 W. L. Murray III and N. L. Key. This is an open access article distributed under the Creative Commons Attribution License, which permits unrestricted use, distribution, and reproduction in any medium, provided the original work is properly cited.

\begin{abstract}
Blade row interactions in turbomachinery can lead to blade vibrations and even high cycle fatigue. Forced response conditions occur when a forcing function (such as impingement of stator wakes) occurs at a frequency that matches the natural frequency of a blade. The objective of this research is to develop the data processing techniques needed to detect rotor blade vibration in a forced response condition from stationary fast-response pressure transducers to allow for detection of rotor vibration from transient data and lead to techniques for vibration monitoring in gas turbines. This paper marks the first time in the open literature that engine-order resonant response of an embedded bladed disk in a 3-stage intermediate-speed axial compressor was detected using stationary pressure transducers. Experiments were performed in a stage axial research compressor focusing on the embedded rotor of blisk construction. Fourier waterfall graphs from a laser tip timing system were used to detect the vibrations after applying signal processing methods to uncover these pressure waves associated with blade vibration. Individual blade response was investigated using cross covariance to compare blade passage pressure signatures through resonance. Both methods agree with NSMS data that provide a measure of the exact compressor speeds at which individual blades enter resonance.
\end{abstract}

\section{Introduction}

Blade row interactions in turbomachinery can lead to blade vibrations and even high cycle fatigue. Forced response conditions occur when the frequency of the forcing function matches the frequency of the blade vibration mode. Forcing functions include the viscous wakes shed from upstream blade rows and the potential fields generated by the upstream and downstream blade rows. It is impossible to remove all resonant crossings from the entire operating range of a multistage axial compressor, and thus, it is important to detect blade vibration to ensure it is within proper limits and to bring to light unexpected engine-order resonant conditions. Since recent turbomachinery designs have moved toward higher pressure ratios and integrally bladed rotors (IBRs), aerodynamic forcing environments have grown in strength and mechanical system damping has decreased. It is now more important than ever to develop robust, dependable methods to detect, characterize, and help mitigate potential vibrational issues before they can have disastrous effects.
Common techniques for detecting blade vibration include strain gauges or tip timing probes. Strain gauge techniques can be employed in a number of ways, but the most common technique involves mounting strain gauges on the blades, and when rotor vibrations are considered, a slip-ring is needed to transmit the signals from the strain gauges to the recording equipment. This measurement is easier if, instead, the strain gauges are mounted to stationary hardware, such as a stator vane or used in a cascade experiment, as done by Freund et al. [1]. Tip timing systems, or nonintrusive stress measurement systems, (NSMS) utilize a set (typically 8 ) of light probes that are arranged in an optimal configuration to detect a particular vibrational mode. Thus, they are limited in the quantity modes that can be measured, and if there is little deflection in the outer part of the blade for the mode of interest, they will not provide any useful information.

Some researchers have also used pressure transducers to study blade vibrations. Pressure transducers can be mounted either on a stationary part of the machine, most often 
the casing endwall as in the work conducted by Baumgartner et al. [2], or they can be mounted onto the rotating hardware, for example Gill and Capece [3], where the signal would be transmitted through a slip ring device to a recorder. When measuring rotor vibrations in the stationary reference frame, the vibration frequency will be Doppler shifted as discussed by Mengle [4] and Kurkov [5].

The acoustic environment inside of an operating compressor is noisy and usually dominated by flow physics unrelated to compressor vibration. Baumgartner et al. [2] used a single hot film downstream of the tip of a vibrating rotor blade and some casing-mounted pressure transducers to investigate rotating stall and flutter vibration of rotating blades. They found that the spectral magnitudes of the instability and vibration were significantly smaller in amplitude than the spectral magnitude related to blade passing frequency. Kurkov [5] showed that the spectrum of the signal obtained when the blade was not vibrating could be subtracted from the spectrum of the pressure signal measured during the forced response vibration to view the frequency spectrum of forced response. This method has also been employed by Mengle [4] in his attempt to remove integral engine order frequencies from observed spectra of a rotating compressor blade. Kurkov [5] was able to take an average frequency response of 16 revolutions of data without vibration and subtract that frequency spectrum from the vibratory response spectrum.

Rotating stall and flutter have been studied extensively in the realm of aeromechanics. These non-EO vibrations tend to have larger vibration amplitudes and correspondingly larger pressure wave amplitudes, making them easier to measure with pressure transducers. Leichtfuss et al. [6] measured non-engine-order stall flutter spectral signatures with stationary Kulite pressure transducers. Schoenenborn and Breuer [7] measured torsional vibration and flutter of blades during surge and were able to detect aeroacoustic signatures from unsteady pressure transducers related to blade motion relatively easily. They were also able to relate the pressure measurements to NSMS tip timing blade deflection data.

The development of techniques that allow pressure transducers to detect forced response during compressor operation as a real-time diagnostic tool or simply as a backup to more complex vibration detection instrumentation is valuable. Pressure fluctuations due to engine order (EO) vibrations such as forced response are typically smaller in comparison to pressure fluctuations due to non-EO vibrations (such as those associated with flutter, rotating stall, and buffeting), making forced response detection difficult. Additionally, forced response pressure fluctuations are Doppler shifted to blade pass frequencies (and their harmonics), which are already heavily influenced by aerodynamics even when the blades are not vibrating, making the identification of forced response pressure components difficult.

The authors are not aware of any open litureature that discussses directly measured engine-order forced response through the use of stationary pressure transducers in an experimental compressor facility, as the amplitudes of the acoustic waves are generally very small in comparison to

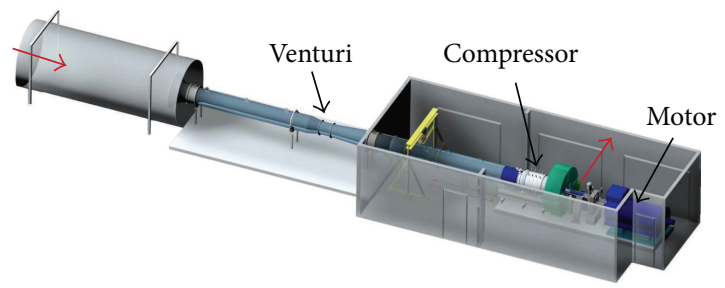

Figure 1: Layout of the Purdue 3-Stage Axial Compressor Research Facility.

other flow field features. Most research conducted to this point has been focused on flow measurements associated with nonintegral engine order vibrations, such as stall, flutter, surge, or acoustic resonances. Fridh et al. [8] measured the spectral component of forced response from partial admission inlet distortion in a turbine using pressure transducers in a rotating reference frame. They were able to detect resonance at Campbell diagram crossings with strain gauge and pressure transducer data. However, the strain gauge data provided more detailed spectral data on the response characteristics of important Campbell crossings compared to the pressure data. Therefore, the objective of this paper is to show how embedded rotor vibrations associated with forced response have been detected with stationary Kulite pressure transducers and describe in detail the important aspects of the instrumentation and data processing techniques that made this possible. This paper marks the first time in the open literature that engine-order resonant response has been measured in a compressor using stationary pressure transducers.

\section{Materials and Methods}

The Purdue Three-Stage Research Compressor is a unique research facility that models the rear stages of a highspeed compressor, matching Mach number and Reynolds number to aircraft engine operating conditions. The facility is conducive to detailed flow measurements in the pitch wise direction because each vane row can be individually indexed past stationary probes. There is a significant and measurable total pressure rise per stage, and blade heights are $50.8 \mathrm{~mm}$, allowing sufficient space for detailed flow measurements without probe blockage issues.

The facility layout is shown in Figure 1. Unconditioned ambient air is drawn into a large settling chamber. Air enters the inlet duct through a bellmouth, which is followed by a series of flow straighteners. An ASME-standard long-form Venturi flow meter installed in the inlet ducting measures the mass flow rate through the compressor. Following an additional length of insulated ducting inside the test cell, a nosecone directs the flow into the annulus of the compressor. The compressor has a constant-area annulus with a $609.6 \mathrm{~mm}$ outer diameter. After passing through the compressor, the air encounters a sliding-annulus throttle and exhausts to atmosphere through a collector. The compressor is driven by a $1 \mathrm{MW}$ AC motor with a variable frequency drive. The motor 


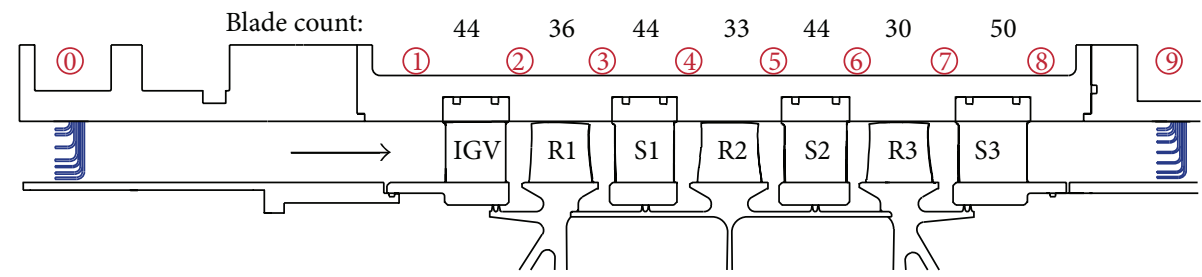

FIGURE 2: A cross-section of the flow path including data acquisition stations.

is connected to a speed-increasing gearbox via a gear coupling to provide the compressor design speed of 5,000 RPM.

The compressor consists of an inlet guide vane (IGV) row followed by three stages, Figure 2. The compressor features IBRs with rotor counts of 36 for Rotor 1, 33 for Rotor 2, and 30 for Rotor 3 . The rotors are attached to a drum in a fixed configuration. The shrouded stator rows have similar vane counts of 44 except for S3, which has 50 vanes. The vane rings are split into two halves and installed in the split casing. Steady compressor performance is measured with 7element Kiel head total pressure and total temperature rakes. The overall compressor total pressure ratio is measured with rakes positioned at Stations 0 and 9.

Figure 3 shows the Campbell diagram for Rotor 2. With 44 vanes upstream in Stator 1 and 44 vanes downstream in Stator 2, the 44 engine order excitation of the first torsion (1T) vibratory mode is the Campbell diagram crossing studied in this research. The frequency of the $1 \mathrm{~T}$ mode is $2700 \mathrm{~Hz}$, and the $44 \mathrm{EO}$ excitation of the R2 1T vibratory mode occurs near 3700 RPM (74\% speed). Also shown is the first torsion mode shape and nodal line as calculated by Fulayter [9] using a finite element analysis.

Tip timing data were acquired to characterize the Rotor $21 \mathrm{~T}$ vibratory response to the $44 \mathrm{EO}$ excitation by measuring the rotor tip deflection. These measurements can be used to compare the results obtained with the new data processing technique developed for the pressure sensors. The Agilis nonintrusive stress measurement system (NSMS) consists of 8 fiber-based optical probes, laser and detector boxes, and an NI 5112 ADC data acquisition chassis. The laser module generates a signal that is sent to each of the 8 probes that shine down on the passing blades. The casing-mounted probes also have a sensing optical cable that transmits the reflection of the blade tips to a photo detector and a pulse-to-digital converter. The converter has a digital clock to track the time of the blade passing and relate it to a $1 / \mathrm{rev}$ shaft signal. The timing of each blade arrival and the correlation of arrival times over all 8 sensors allow the measurement of the amplitude and phase of vibration for each blade.

The resolution of the tip timing measurements is a function of the counter timer board clock speed, the rotor tip diameter, and the rotor tip speed. With a sampling period of 2 microseconds, the resolution of the tip timing data near the 1T vibratory resonance of Rotor 2 is about 0.009 mils. Since it is unsafe to operate at the resonance speed due to potential fatigue and failure of the vibrating rotor, the data are acquired during a transient speed change through the resonant condition, and the sweep rate is an important

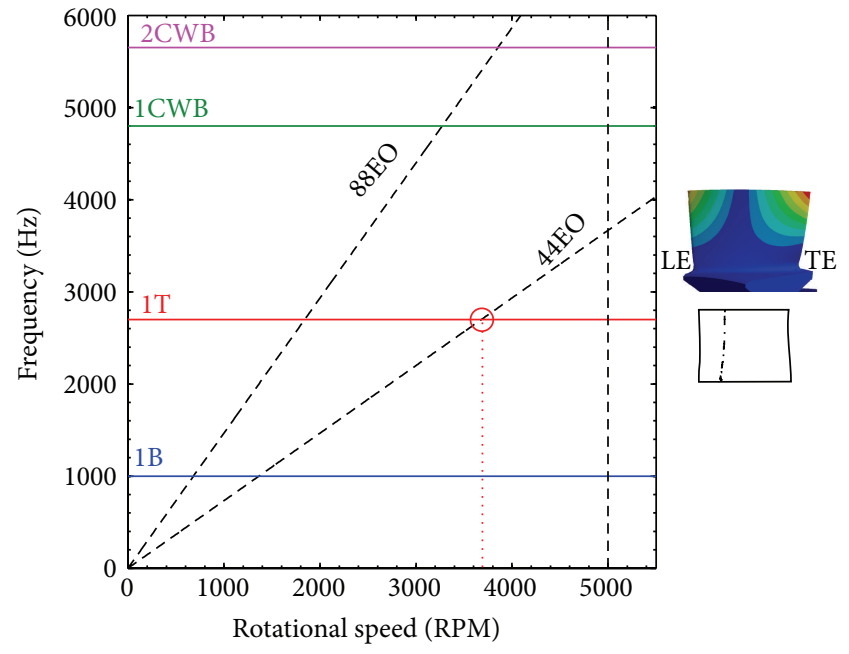

FIGURE 3: Campbell diagram for Rotor 2 including mode shape and nodal line for the $1 \mathrm{~T}$ vibratory mode.

parameter. As suggested by von Flotow [10], the critical sweep rate is defined as the ratio of the half-power bandwidth acquired over 3 vibration cycles. For the $1 \mathrm{~T}$ resonance of Rotor 2, the critical sweep rate is around $15 \mathrm{rpm} / \mathrm{s}$. Also, the control of the compressor drive system was programmed such that the compressor speed would have a constant acceleration rate through the resonant condition, and thus, this aspect of the experiment was fully controlled. Sweep rates as low as $2.2 \mathrm{RPM} / \mathrm{s}$ were utilized.

The steady loading of the compressor was adjusted using the throttle. Two loading conditions were considered: nominal loading (NL) and high loading (HL). Figure 4 shows the compressor performance map including two speedlines: $68 \%$ and $80 \%$ corrected speed. These speedlines bound the resonant condition studied. Each point on the speedline represents the area-averaged pressure ratio as determined from a vane traverse every $5 \%$ passage to include the effects of the vane wakes. The mass flow rate has been normalized by the stall flow rate at $68 \%$ corrected speed. The total pressure ratio is the overall compressor total pressure ratio. The operating lines were created by quickly scanning the pressure rakes and flow meter while accelerating the compressor through resonant speed, and thus, they are not area-averaged quantities.

The pressure measurements were acquired from the downstream vane which was instrumented for a different research project. The sensors were Kulite LQ-062 pressure 


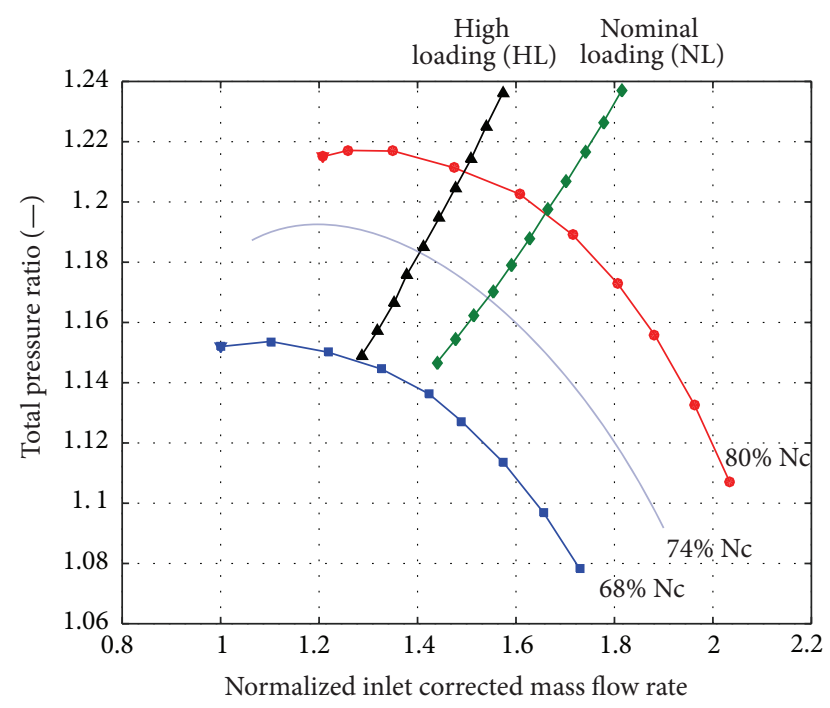

FIGURE 4: Compressor operating map including operating lines.

transducers with a range of $0-5 \mathrm{psig}$. The key aspect that made these sensors useful in blade vibration detection is that they had no screen, and this resulted in a high frequency response, as much as $100-150 \mathrm{kHz}$ per manufacturer's specifications. Other sensors in the facility with lower frequency response did not capture the same phenomenon. To briefly describe the installation of these sensors, a removable S2 passage that could be sent to Kulite for sensor installation was fabricated, Figure 5. The part was constructed of 17-4 PH stainless steel and EDM cut into 3 segments. The vanes of the removable passage were machined to accommodate a total of 16 transducers, 8 on each vane. They were positioned at $50 \%$ span and $80 \%$ span, and axial positions include $10 \%, 20 \%$, $30 \%$, and $40 \%$ chord on the pressure side of one blade and the suction side of another. The sensors were clustered as close as possible to the leading edge, and RTV was used to back fill the pockets drilled for the sensors.

Figure 6 shows a diagram of the location of the pressure transducers installed in Stator 2 with respect to the flowpath. Because of space restrictions, a single vane could not support transducers on both the pressure side and suction side. Thus, a passage was instrumented, with the transducers measuring a particular flow passage. This arrangement was also favored because RTV was used to backfill the pocket, and thus, the RTV did not affect the pressure measurements since measurements were not made on the same surface with the RTV treatment.

Figure 7 shows photographs of the removable Stator 2 insert with the Kulite pressure transducers installed. The wires are fed through a hole in the casing and the vane row is not traversed when this passage is installed. In the photograph on the right, the RTV on the suction side of the top vane is visible. The unsteady pressure on the pressure side of the top vane is measured. Also, some of the holes for the pressure measurements on the suction side of the bottom vane are visible.

The excitation signal and amplification for the Kulites were provided by a Precision Filter 28000 chassis with quantity 4, 28118 full bridge amplification cards, each capable of amplifying 8 channels. Data were digitized with an NI PXI-1073 chassis with two 16-channel NI PXIE-6358 cards, with a total bandwidth of $1.25 \mathrm{MS} / \mathrm{s}$ per channel for all 32 channels simultaneously sampled. The transducers were calibrated with a calibration chamber, Figure 8, constructed from $15.24 \mathrm{~cm}$ cast aluminum pipe that was $15.24 \mathrm{~cm}$ long with caps on each end. The stator was installed in the chamber by a short piece of threaded rod that was threaded into one of the machined and tapped threads in the shroud of the stator. The LEMO connectors were fed out of two holes drilled in one of the end caps and sealed with silicone sealant. The heat shrink used on the outside of each of the wires was also sealed at each end with silicone to keep air from leaking through the heat shrink and out of the calibration chamber. The slope of the calibration for the installed stator sensors was as much as $25 \%$ different from the values quoted by Kulite for the sensors prior to installation in the stator highlighting the need for calibration.

\section{Results and Discussion}

Since this is a research compressor that operates at lower pressure levels than an actual gas turbine compressor, the pressure waves associated with blade vibration are expected to be small. To assess the ability of the available pressure instrumentation to detect the blade vibration, a simple analysis was performed. LINSUB, a linear, flat plate cascade, aeromechanics solver created by Whitehead [11], was used to predict the expected pressure amplitude due to the vibration of Rotor 2 for this forced response condition. It outputs the absolute and fluctuating components of the flow field (such as lift, pressure, and moments) related to bending, torsion, chord wise bending, and wakes/gusts. In this case, it is of interest to calculate the unsteady pressure waves traveling upstream and downstream from a blade vibrating in torsion. Based on the compressor geometry and previously measured flow conditions near resonance, LINSUB predicted an upstream-traveling pressure component of $292.3 \mathrm{~Pa}$ and a downstream-propagating pressure wave of amplitude 584.7 Pa.

The typical uncertainty of the Kulite pressure transducers model LQ-062 is $\pm 0.1 \%$ of the full range of the transducer, which corresponds to $\pm 34.5 \mathrm{~Pa}$. (The manufacturer provides a typical and maximum uncertainty, where the maximum is $\pm 0.5 \%$.) This uncertainty does not include any uncertainty or noise introduced by connectors, cables, Precision Filter amplifier equipment, or the PXI analog-to-digital conversion. By including these effects, the largest resulting uncertainty in the Kulite pressure measurements amounts to approximately $\pm 175.8 \mathrm{~Pa}$. This was calculated considering that the Precision Filter 28118 amplifier cards have an accuracy of $\pm 0.1 \%$ of full scale range and the PXI-6358/6356 A/D converters have an uncertainty of $0.012 \%$ full scale.

The LINSUB analysis is $1 \mathrm{D}$, and thus serves as an approximation of the expected pressure amplitudes due to rotor vibration. Nonetheless, the results indicate that at these compressor speeds, the pressure waves due to resonance are small. However, if signal processing techniques can be 


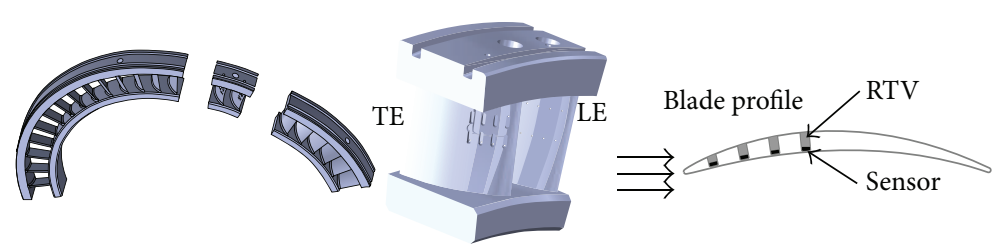

FIGURE 5: Vane ring modification for accommodation of Kulite sensors.
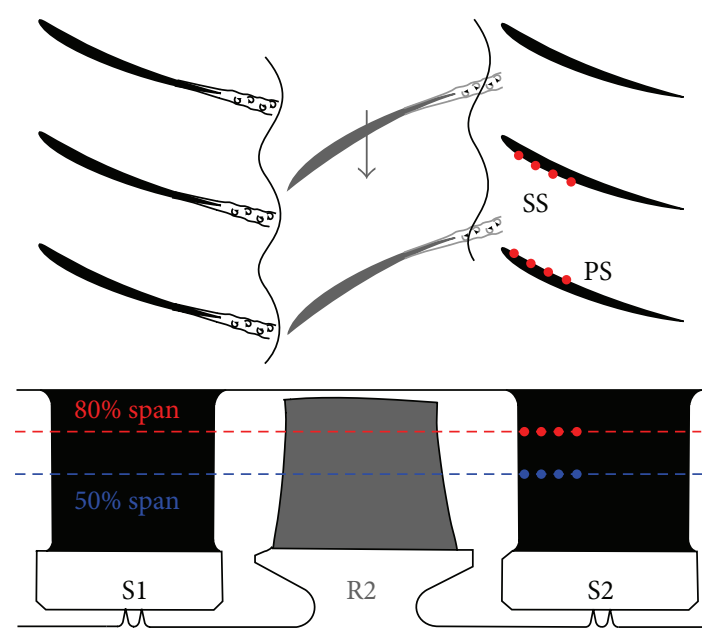

FIGURE 6: Top-down and side view of Stator 2 with installed pressure transducers.

developed to identify the blade vibration effects at these low compressor speeds, then vibrations in actual compressors which operate at higher speeds should be easier to detect.

To detect these small pressure waves generated by the vibrations of Rotor 2 , several data processing methods were investigated. The most difficult issue was the removal of background noise and pressure information that was not pertinent to the blade vibration. Additionally, data handling algorithms to manage the large volumes of unsteady binary data were developed.

The Kulite pressure transducers are in the stationary reference frame, but the vibrating rotor is in the relative reference frame, and thus, the frequency of vibration as measured by the transducer will be shifted due to the relative motion between the rotor and the transducer. Mengle [4] showed that since the rotor is spinning at a rotation rate, $\Omega$, the vibration frequency of the blades, $\omega$, will be Doppler shifted to a different frequency, $\omega^{\prime}$, based on nodal diameter, $\mathrm{ND}$, and wave number, $m$, in (1).

$$
\omega^{\prime}=\omega+(\mathrm{ND}+m B) \Omega .
$$

The nodal diameter can either be determined from the NSMS data or from the difference between stator vanes and rotor blades, since the frequency of vibration is an engineorder (EO) forced response, in this case, as shown in

$$
\mathrm{ND}=N_{\text {Stator Vanes }}-N_{\text {Rotor Blades }}=44-33=11 .
$$

Because of blisk mistuning, the approximate band of blade response varies over a range of $2700-2735 \mathrm{~Hz}$, as measured with NSMS. By choosing $m$ values of $-2,-1,0,1$, and 2 , the first few wave modes will be captured. These should be the highest responding modes. When the frequency of interest is associated with an engine-order forced response vibration, the Doppler shift will always shift the forced response to multiples of the blade pass frequency. Therefore, the signal processing challenge is to determine changes in the $33 / \mathrm{rev}$ frequency (and higher harmonics) in the spectrum. This may prove difficult because as the compressor rotational speed increases, changes in the strength of these components of the signal will also be associated with aerodynamic changes. However, if a spike in the higher harmonics of the signal is visible only in a RPM band where rotor vibration is known to occur (as measured with the tip timing data), then it will confirm that the pressure sensors detected rotor vibration.

An effective way to analyze the change in the pressure spectrum with time is with Fourier transform waterfall graphs. A cartoon explaining the construction of these graphs is shown in Figure 9. The pressure data from the sensors are recorded as the compressor rotational speed is increased at a constant rate through the speed corresponding to the first torsion vibratory mode. First, the pressure data from a particular channel are divided up into separate compressor revolutions using the use of the once-per-revolution signal from the compressor shaft. Simply dividing the data by each revolution is effectively square windowing the signal, which can introduce unwanted artifacts into the frequency spectrum, and thus, a Kaiser window was applied to each revolution. A Kaiser window allows a high resolution between two frequency components that are separated greatly in amplitude but closely related by frequency. This windowing technique allows for the detection of small components of the $1 \mathrm{~T}$ signal that are Doppler shifted to a frequency slightly different than blade pass frequency due to individual blade mistuning. It also allows for a cleaner waterfall plot, resolving features due to a better resolution in frequency.

After windowing the revolution of data, a Fourier transform is performed, and the frequency is normalized by the rotor blade pass frequency. This normalization is important because it connects the spectral magnitudes to blade pass events rather than to physical compressor speeds, which are constantly changing through the sweep. Pressure fluctuations will occur with the blade passing events due to rotor wakes impinging on the downstream vane row. Thus, a significant magnitude in the spectral component associated with blade pass frequency will always be present. To reduce this effect in the hopes of identifying the small pressure waves associated 


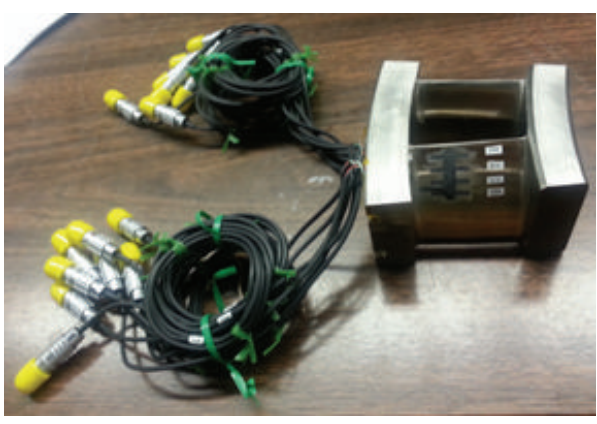

(a)

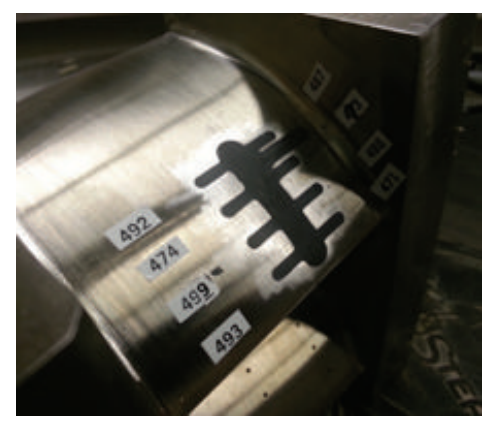

(b)

FIGURE 7: Photographs of the removable Stator 2 insert with pressure transducers installed.

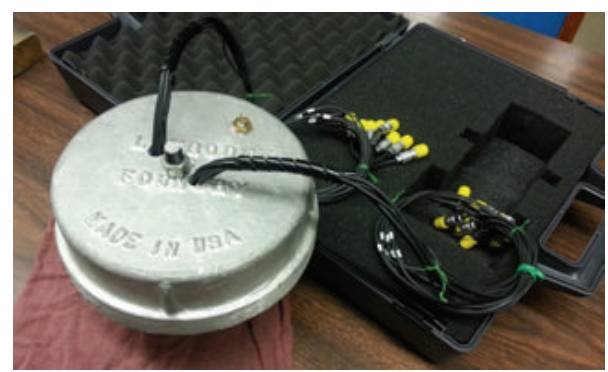

(a)

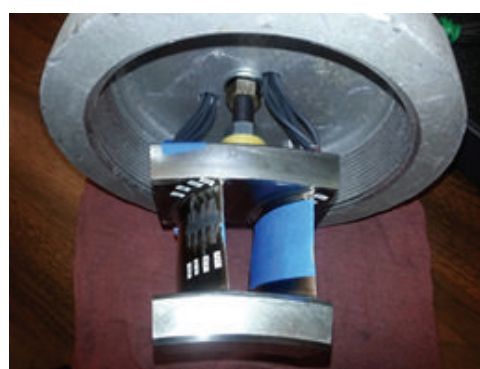

(b)

FIGURE 8: Calibration chamber setup for the vane-mounted pressure transducers.
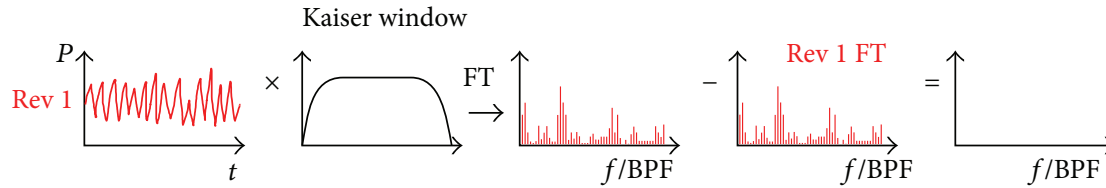

$|\mathrm{FT}(\operatorname{Rev} i)-\mathrm{FT}(\operatorname{Rev} 1)|$
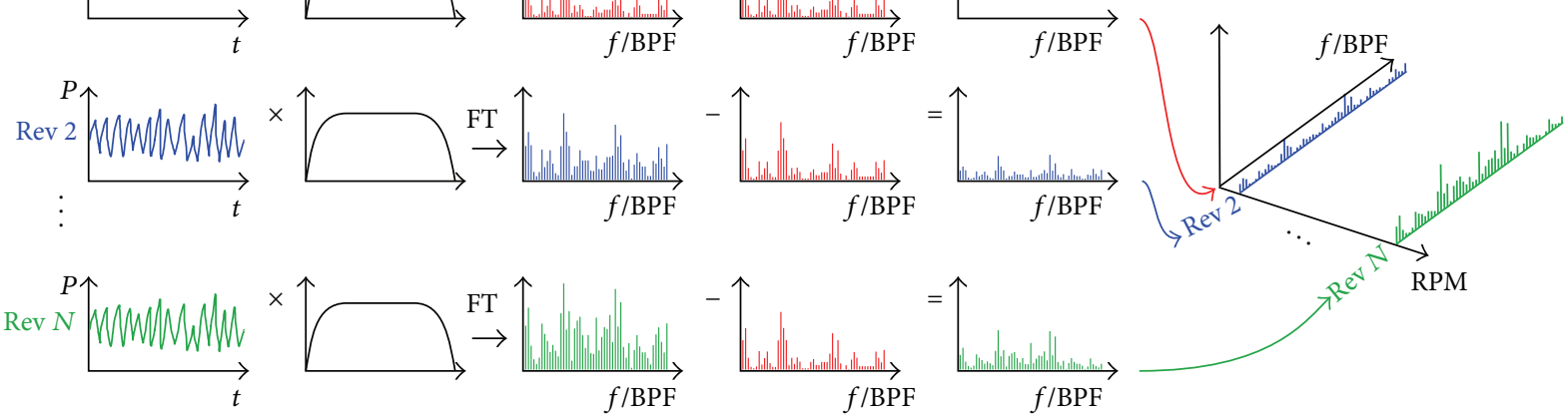

FIGURE 9: Data processing strategy for generating waterfall graphs.

with the resonant vibration, the spectral magnitudes measured at the beginning of the sweep ( $3670 \mathrm{rpm}$ ) are subtracted from the spectra at all successive revolutions. Then, these reduced spectra are assembled to create the resulting waterfall graph. This provides a snapshot of several thousand Fourier transforms, allowing trends to be easily identified. This also allows for easy comparison of the changes in amplitude of a given frequency band as the compressor accelerates through resonance.

Figure 10 shows the waterfall graph constructed from data acquired at nominal loading. The labels on the right show particular blade pass frequencies and their harmonics. Recall the blade counts decrease by 3 (Rotor 1 has 36 blades, Rotor 2 has 33 , and Rotor 30 has 30 ). The $44 \mathrm{EO}$ excitation of the Rotor 2 first torsion vibration should occur at Rotor 2 blade pass frequency (33/rev) or its harmonics. The $33 / \mathrm{rev}$ frequency component is, for the most part, nonexistent. Also, the 66/rev tends come and go. However, the $99 / \mathrm{rev}$ either is nonexistent or appears and disappears in conjunction with resonance. There are also other frequencies present, namely, responses at 30/rev, 36/rev, higher order harmonics of these frequencies (which correspond to R1 and R3 blade pass frequencies). This is due to the pressure transducers picking up the destructive and constructive interference of R1 and R3. The manner in which the reflected multiples of these blades pass frequency harmonics is transmitted through the compressor and could 


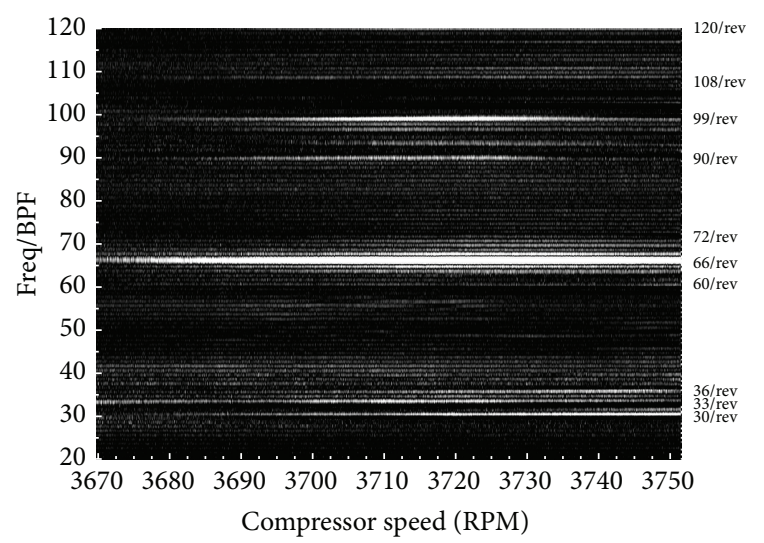

FIGURE 10: Waterfall graph at nominal loading.

be indicative of resonance, perhaps even R3 resonance which occurs in the speed range of 3705-3805 RPM.

Figure 11 shows the amplitude of the $99 / \mathrm{rev}$ response (taken to be the sum of the amplitudes in the frequency band between 98.5/rev and 99.5/rev), and the response grows and decays as a function of compressor speed. For comparison to NSMS data, Figure 12 shows the RPM band of the Rotor 2 resonant response. Each blade has its own natural frequency, and the results from all 33 blades are overlaid. One graph shows the results at nominal loading and the other at high loading. The mean loading does not change the frequency of the response, but it does change the amplitude of the response. The compressor speeds (or frequency) of the $1 \mathrm{~T}$ response ranges from approximately 3700 to $3730 \mathrm{RPM}$. The range at which the $99 / \mathrm{rev}$ frequency bands increase in amplitude from the pressure transducer measurements agrees well with the NSMS data.

The 66/rev response grows but then seems to level out as RPM is increased, and this could be attributed to the data processing technique, where the nonvibrating spectrum at the low speed range of the sweep was used to adjust the other spectra, as shown in Figure 9. Therefore, if the spectral magnitudes change because of the change in compressor speed resulting in a change of the aerodynamic interaction of the blade rows, then that would appear in the waterfall graphs as well.

The 33/rev spectral component does not have a significant amplitude, and this is a result of the data processing methods. The blade pass frequency component is large and by subtracting the spectrum from the first revolution off of all subsequent signals, it was sufficient to remove the blade pass component from the other revolutions. The spectral magnitude of blade pass frequency was an order of magnitude higher than the harmonics, and thus, it would be difficult to identify the small pressure fluctuations associated with rotor vibration at this frequency. Therefore, to detect forced response vibration effects from the vane pressure signal, efforts should focus on the higher harmonics of blade pass frequency where the Doppler shifted forced response frequency components are on the same order of magnitude as the amplitudes that occur when the blade is not vibrating.

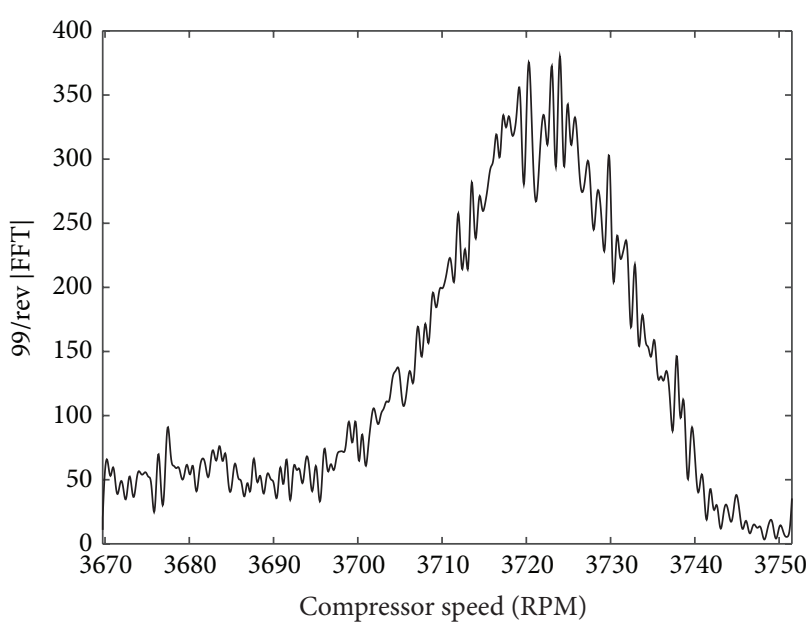

FIGURE 11: The magnitude of the spectral component associated with the $99 /$ rev frequency at nominal loading.

The waterfall graph for the results at high loading is shown in Figure 13. As before, the magnitude of the $99 / \mathrm{rev}$ spectral component signal is shown in Figure 14. The increased spectral magnitude of the $99 /$ rev frequency occurs over the same speed range as the NSMS system measured the rotor vibration, as shown in Figure 12. The 33/rev and 66/rev frequencies in the waterfall graph follow the same trends as shown at nominal loading: the $33 / \mathrm{rev}$ contribution has been subtracted out and the $66 / \mathrm{rev}$ contribution seems to be increasing with compressor speed.

In summary, the frequency domain analysis showed that the pressure transducers did detect the rotor vibration at a Doppler shifted frequency associated with 3rd harmonic of blade pass frequency $(99 / \mathrm{rev})$. This waterfall analysis considers the spectrum on a revolution-by-revolution basis. Another way to investigate these data is to focus on a time domain analysis where individual blade pass events are considered, rather than a full revolution of data. The advantage of having NSMS data is the vibration frequency and amplitude of each rotor blade is recorded, and thus, the pressure signals associated with highly responding blades can be investigated. This can be done with a cross correlation which essentially looks for a blade pass event that appears to be different than the others. The pressure trace measured when the blade is vibrating will be different than that measured when the blade is not vibrating, and thus a low correlation value could be an indicator of the forced response event.

Cross correlation and cross covariance are methods of determining the "similarity" or likeness, of two random vectors or signals, denoted by $x$ and $y$ with length $N$. By varying the offset, $m$, between the two vectors, a correlation sequence of the two vectors is generated and is a function of the element-by-element product of each point in the vector, as shown in

$$
\widehat{R}_{x y}(m)= \begin{cases}\sum_{n=0}^{N-m-1} x_{n+m} y_{n}^{*}, & m \geq 0 \\ \widehat{R}_{y x}^{*}(-m) & m<0 .\end{cases}
$$




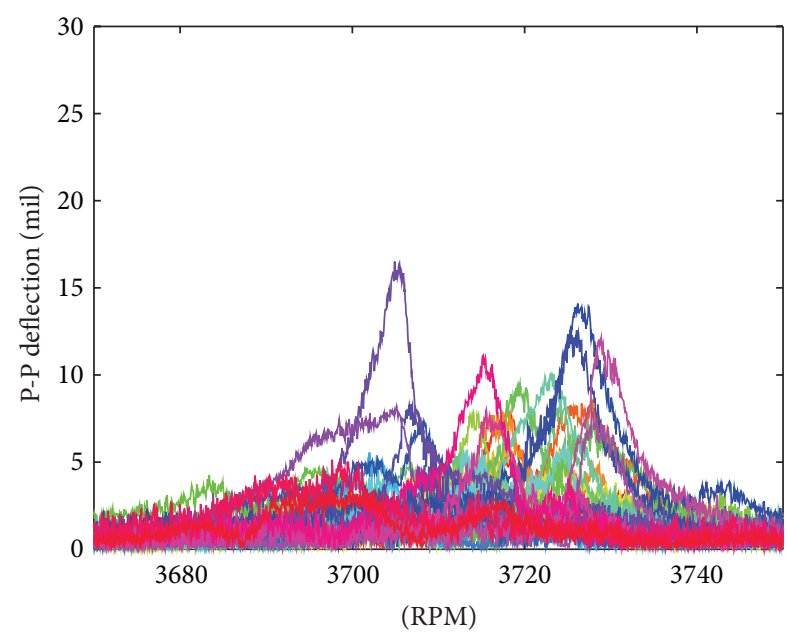

(a)

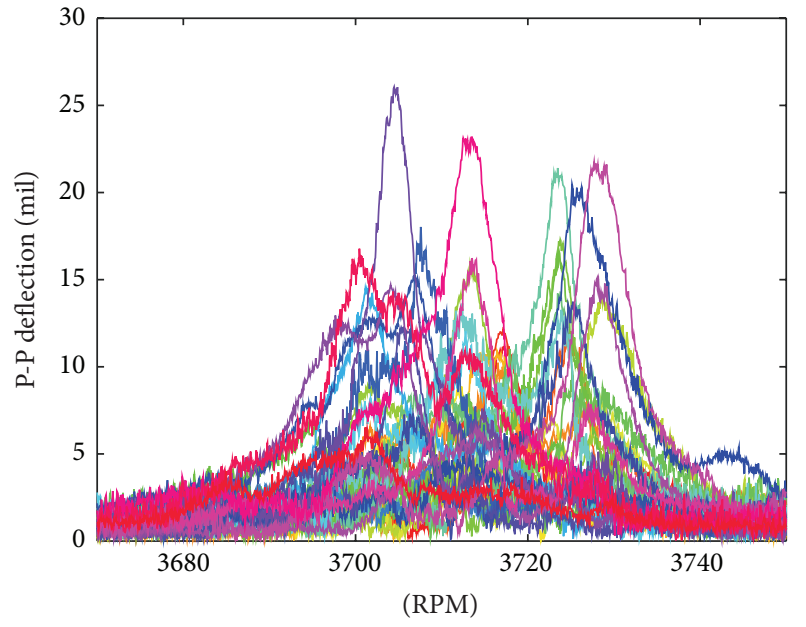

(b)

FIGURE 12: NL (a) and HL (b) peak-to-peak deflection of each blade of R2 as recorded by NSMS.

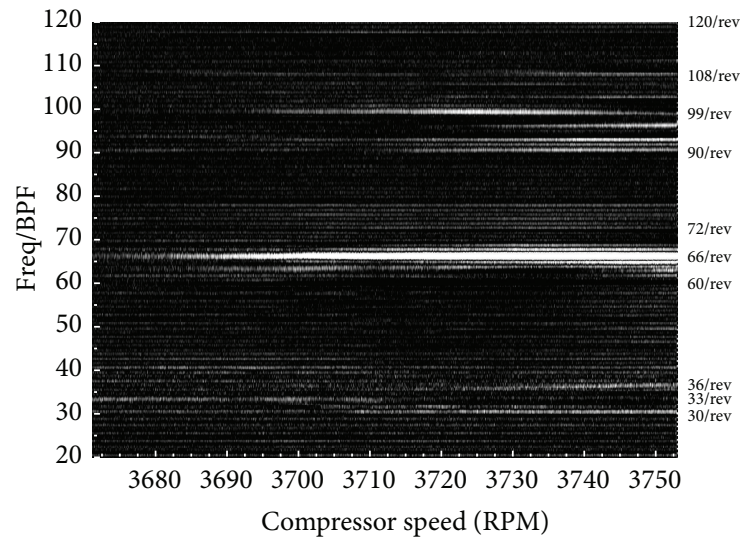

FIGURE 13: Waterfall graph at high loading.

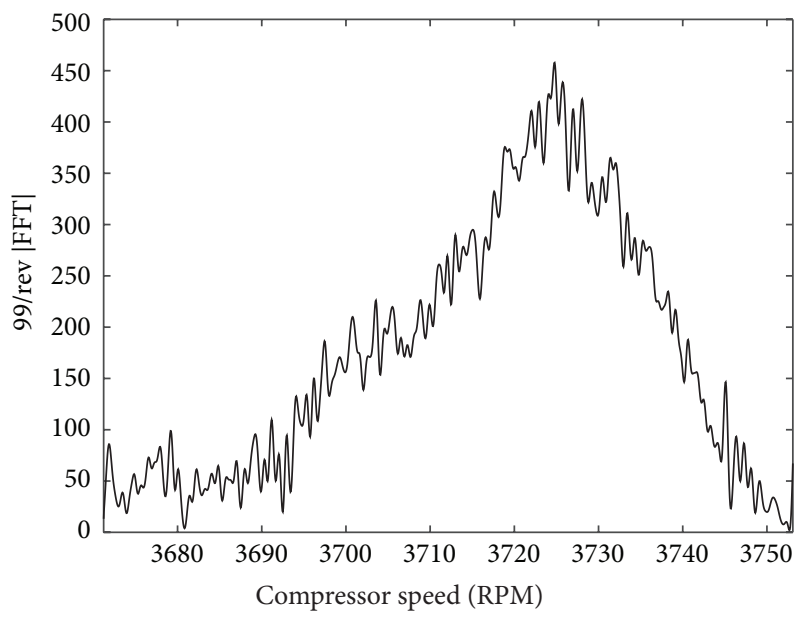

FIGURE 14: The magnitude of the spectral component associated with the $99 /$ rev frequency at high loading.
Cross correlations, however, are not normalized, and since the amplitude of the mean level of pressure continually increases due to the increase in compressor total pressure ratio with increasing speed during the sweep, the transient nature of this experiment renders the cross correlation inappropriate. Therefore, to remove the effects of the magnitude differences, the cross covariance is considered. It is the product of two vectors, which are normalized by their mean, denoted by $(1 / N) \sum_{i=0}^{N-1} x_{i}$ and $(1 / N) \sum_{i=0}^{N-1} y_{i}^{*}$. The cross covariance, (4), is used since there will be no overall rise in correlation due to the rise in overall compressor pressure ratio. Consider

$$
\begin{aligned}
c_{x y}(m) & \left\{\begin{array}{cc}
\sum_{n=0}^{N-|m|-1}\left(x(n+m)-\left(\frac{1}{N}\right) \sum_{i=0}^{N-1} x_{i}\right) \\
\times\left(y_{n}^{*}-\left(\frac{1}{N}\right) \sum_{i=0}^{N-1} y_{i}^{*}\right) & m \geq 0, \\
c_{y x}^{*}(-m) & m<0 .
\end{array}\right.
\end{aligned}
$$

Figure 15 shows the pressure traces measured on the pressure side of Stator 2 when Blade 26 was passing in front of it. The different traces are shown for different compressor speeds to illustrate how the pressure signal associated with a particular Rotor 2 blade is changing throughout the compressor sweep. The red trace at 3671 RPM is the pressure trace from the 1st full revolution of data to which all successive pressure traces are compared for this analysis. The blue and cyan lines, at 3705 and 3710 RPM, respectively, show pressure traces of the blade in and around resonance, and the 3735 RPM black lines shows the pressure trace near the end of the sweep. This illustrates how much the shape of the pressure signal will change as the compressor pressure ratio increases.

In Figure 16, there is a large drop in cross covariance around 3705 RPM, which corresponds directly to the RPM 


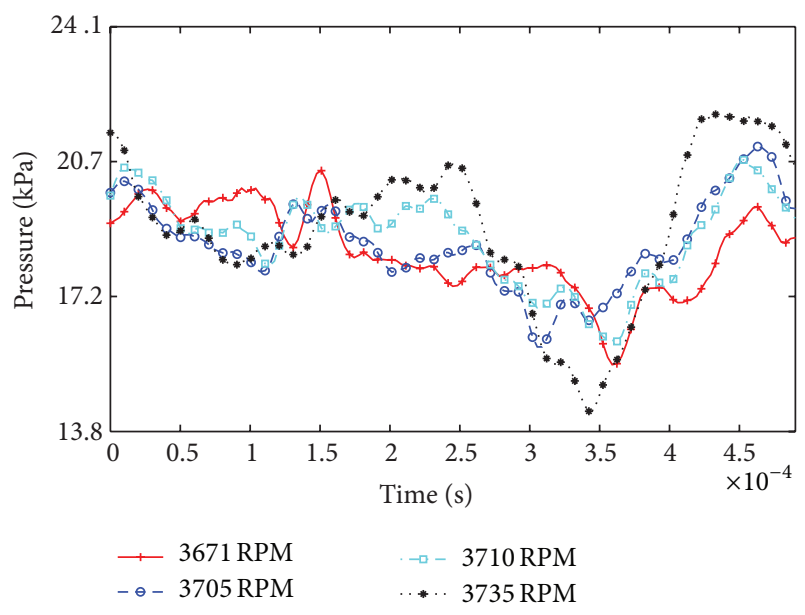

Figure 15: Rotor blade passage traces, Blade 26, PS $80 \%$ Span $20 \%$ Chord, HL.

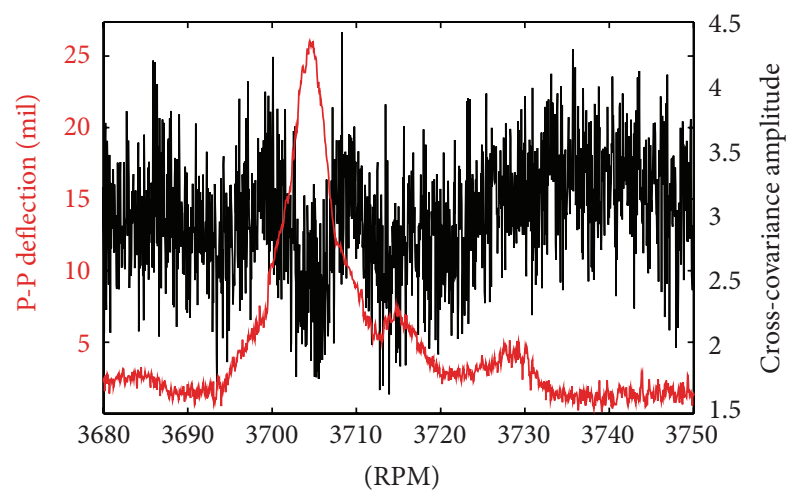

FIGURE 16: Cross covariance and NSMS deflection for Blade 26 at high loading ( $80 \%$ span).

band of maximum displacement in R2 for blade 26 at high loading (refer to Figure 12). Although the drop in covariance amplitude is small in comparison to the revolution-torevolution changes in covariance amplitude (i.e., the noise in the signal), it is apparent that there is approximately a $30 \%$ drop in average covariance amplitude from 3700 to 3705 RPM, and then a corresponding $30 \%$ increase at $3705-$ 3710 RPM.

Blade 22 is another high responding blade according to the NSMS data from Figure 12. The cross covariance of the pressure signal acquired at $80 \%$ span shows a similar drop in cross covariance, Figure 17. The compressor speed range over which this occurs is roughly equivalent to the speed range for which the NSMS data show that the blade vibration occurs. There is an inherent waviness, or fluctuation, in the overall trend of the cross covariance that is apparent in all sensors regardless of speed, which makes the measurements of cross covariance amplitude drops difficult to compute. Future work is aimed at advanced signal processing techniques to further explore the utility of the cross covariance in identifying rotor vibration.

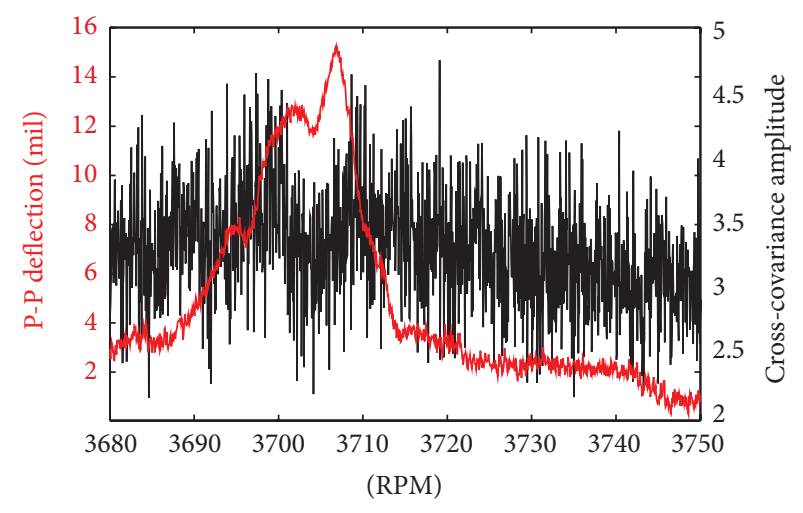

FIGURE 17: Cross Covariance and NSMS deflection for Blade 22.

\section{Conclusions}

This is the first time in the open literature that measurements of a compressor forced response vibrational mode have been detected with stationary pressure transducers. This paper explains the particular data processing methods used to identify rotor vibration. The data processing was challenging since the data were transient in nature; the compressor was accelerated through the resonant speed as the data were collected to avoid depleting several cycles from the life of the blade. An additional challenge was associated with the fact that forced response rotor vibrations always get Dopplershifted to a harmonic of blade pass frequency when measured in the absolute reference frame. This makes it difficult to identify the smaller contributions of that spectral magnitude that are associated with vibrations rather than the aerodynamics of the blade passing event. On top of all this, the smaller pressure levels associated with a research compressor result in small-amplitude pressure waves generated when the blade vibrates. Despite all these challenges, the technique was successful.

Detection of engine-order forced response at part speed compressor operation through the use of stationary pressure transducers could have an impact on the way blade vibrations are measured in turbomachinery, as this measurement method is significantly simpler than other advanced vibration measurements, such as rotating instrumentation and/or laser-based tip timing measurements. It can also revolutionize the health monitoring strategy of gas turbine compressors as it can identify high cycle fatigue issues prior to blade failure.

The focus of this research was to interrogate the signals obtained from fast-response pressure transducers while a 3-stage compressor was being accelerated through force response resonant speeds. The 44 engine order excitation of the first torsion vibrational mode of the embedded Rotor 2 was studied. Kulite pressure transducers without screens had enough frequency response to detect the blade vibration. In this experiment, the transducers of this type were installed in the downstream stator vane, but flushmounting this sensor in the casing over the rotor would 
be even more convenient. LINSUB was used to estimate whether the Kulite pressure transducers would be able to detect acoustic signatures from this forced response event, and it showed that the acoustic pressure fluctuations would be slightly higher (3-4 times) than the uncertainty in the Kulite pressure measurement. The amplitude of the pressure wave will increase with compressor rotational speed so while the pressure signature associated with blade vibration may be small in this research vehicle, it could be more significant at actual engine operating conditions rendering this technique perhaps even more effective for higher speed compressor investigations.

Data processing in both the frequency and time domain show that the acoustic signature generated by the vibrating rotor is detectable. Through the calculation of Dopplershifted vibration frequencies, spectra related to the rotor vibration have been detected in the 3rd harmonic of the rotor blade pass frequency. Additionally, through the use of a cross covariance blade-to-blade similitude analysis, individual blade vibration has been detected and confirmed with NSMS tip timing results.

Future work is aimed at advanced signal processing techniques to further explore the utility of the cross covariance in identifying rotor vibration. In particular, the noise in the cross correlation results must be reduced if this type of signal is to be used in a control loop for compressor operation. Also, close collaboration with sensor manufacturers should be aimed at reducing the cost and increasing the durability of these sensors for implementation in actual gas turbines.

\section{Nomenclature}

$\omega$ : Torsional vibration frequency

$\omega^{\prime}$ : Doppler-hifted torsional vibration frequency

ND: Nodal diameter

$B$ : Number of blades

$m$ : Integer, harmonic number, lag offset

$\Omega: \quad$ Rotational velocity $[1 / s]$

$k$ : Wave number, spring constant

$c_{x y}$ : Covariance term

$N$ : Vector length

$\widehat{R}_{x y}$ : Cross covariance term

$x, y$ : Arbitrary vectors.

\section{Conflict of Interests}

The authors declare that there is no conflict of interests regarding the publication of this paper.

\section{Acknowledgments}

This work was funded by the GUIde IV Consortium, which included NASA, the US Air Force, Rolls-Royce, GE, Pratt \& Whitney, Honeywell, Siemens, and Mitsubishi. The authors are grateful to the GUIde IV Consortium for its guidance and financial support of this work. Also, the authors acknowledge Natalie Smith's artistic contribution to the figures.

\section{References}

[1] O. Freund, M. Bartelt, M. Mittelbach, M. Montgomery, D. M. Vogt, and J. R. Seume, "Impact of the flow on an acoustic excitation system for Aeroelastic studies," ASME Journal of Turbomachinery, vol. 135, no. 3, Article ID 031033, 2013.

[2] M. Baumgartner, F. Kameier, and J. Hourmouzaiadis, "Nonengine order blade vibration in a high pressure compressor," in Proceedings of the ISABE International Symposium on Airbreathing Engines, Melbourne, Australia, September 1995.

[3] J. D. Gill and V. R. Capece, "Experimental investigation of flutter in a single stage unshrouded axial-flow fan," in Proceedings of the 42nd AIAA Aerospace Sciences Meeting and Exhibit, pp. 79978005, Reno, Nev, USA, January 2004.

[4] V. Mengle, "Acoustic spectra and detection of vibrating rotor blades, including row-to-row interference," in Proceedings of the AIAA 13th Aeroacoustics Conference, Tallahassee, Fla, USA, October 1990.

[5] A. P. Kurkov, "Flutter spectral measurements using stationary pressure transducers," Journal of Engineering for Power, vol. 103, no. 2, pp. 461-467, 1981.

[6] S. Leichtfuss, F. Holzinger, C. Brandstetter, F. Wartzek, and H. P. Schiffer, "Aeroelastic investigation of a transonic research compressor," in Proceedings of ASME Turbo Expo, San Antonio , Tex, USA, June 2013.

[7] H. Schoenenborn and T. Breuer, "Aeroelasticity at reversed flow conditions-part II: application to compressor surge," Journal of Turbomachinery, vol. 134, no. 6, Article ID 061031, 8 pages, 2012.

[8] J. Fridh, B. Laumert, and T. Fransson, "Forced response in axial turbines under the influence of partial admission," Journal of Turbomachinery, vol. 135, no. 3, Article ID 041014, pp. 041014-1041014-9, 2013.

[9] R. D. Fulayter, An experimental investigation of resonant response of mistuned fan and compressor rotors utilizing NSMS [Ph.D. dissertation], School of Mechanical Engineering, Purdue University, West Lafayette, Ind, USA, 2004.

[10] A. von Flotow, Hood Technologies Corporation's Analyze Blade Vibration Software Version 3.3 User Manual, 2003.

[11] D. S. Whitehead, "Unsteady two-dimensional linearized subsonic flow in cascades," in AGARD Manual on Aeroelasticity in Axial-Flow Turbomachines, vol. 1, pp. 3.24-3.30, 1987. 

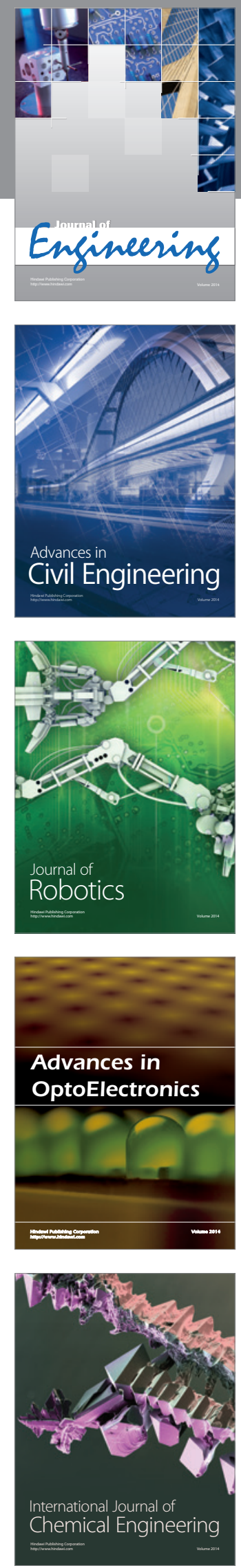

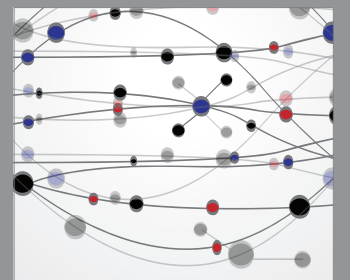

The Scientific World Journal
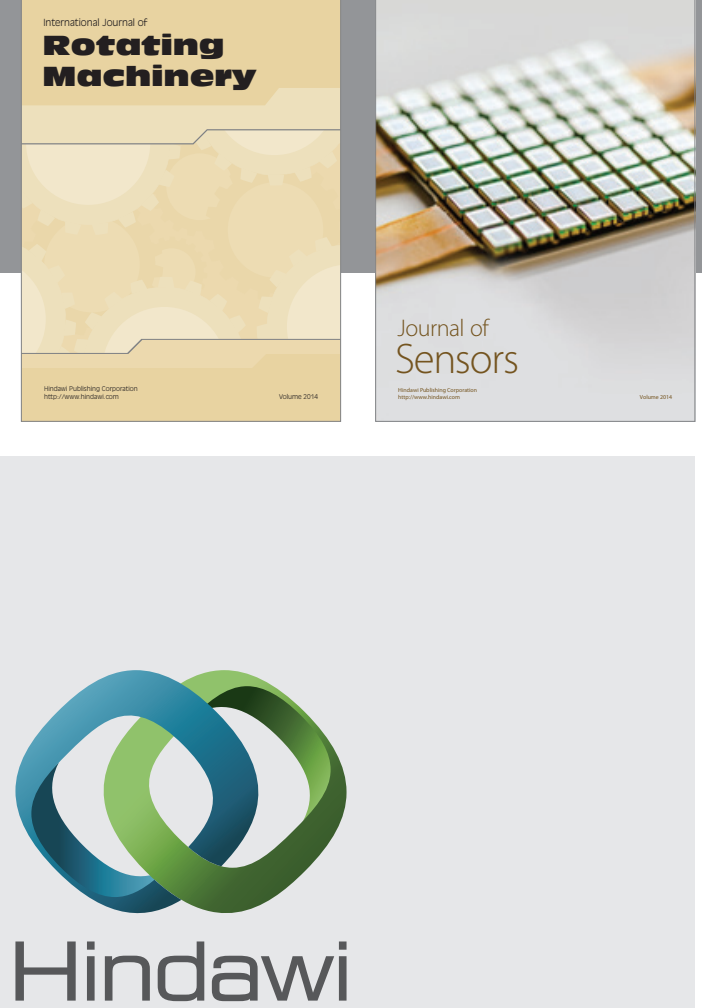

Submit your manuscripts at http://www.hindawi.com
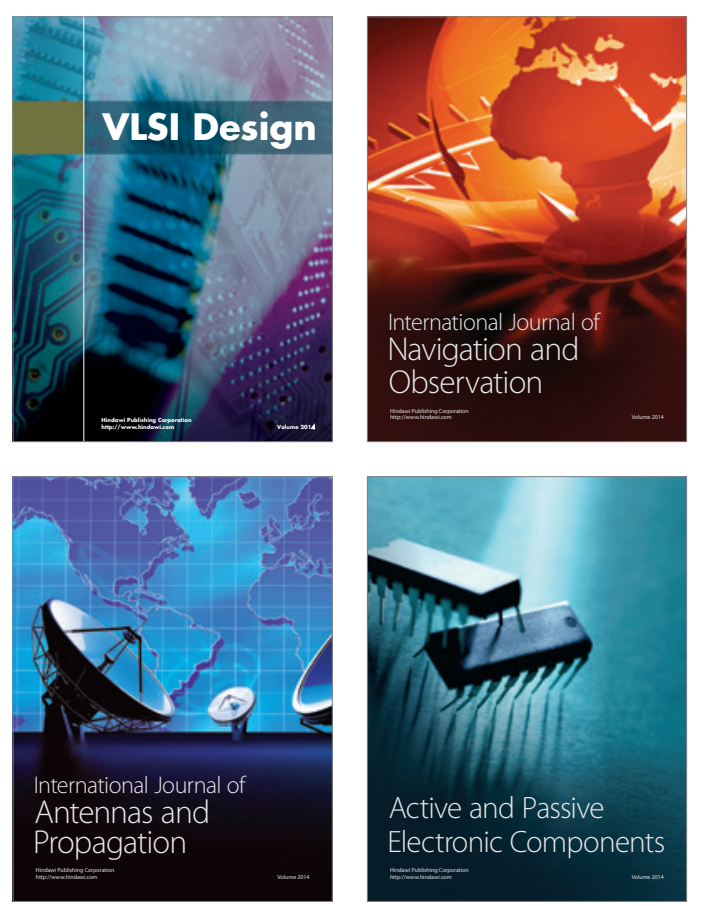
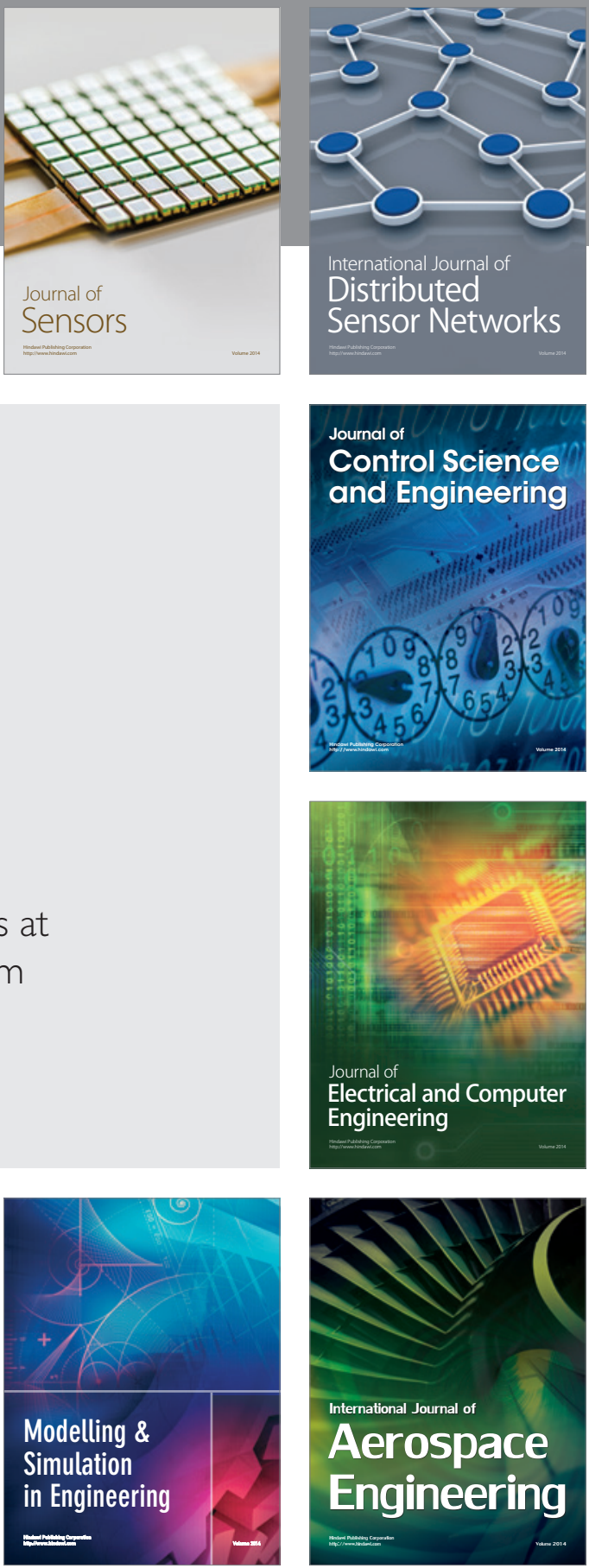

Journal of

Control Science

and Engineering
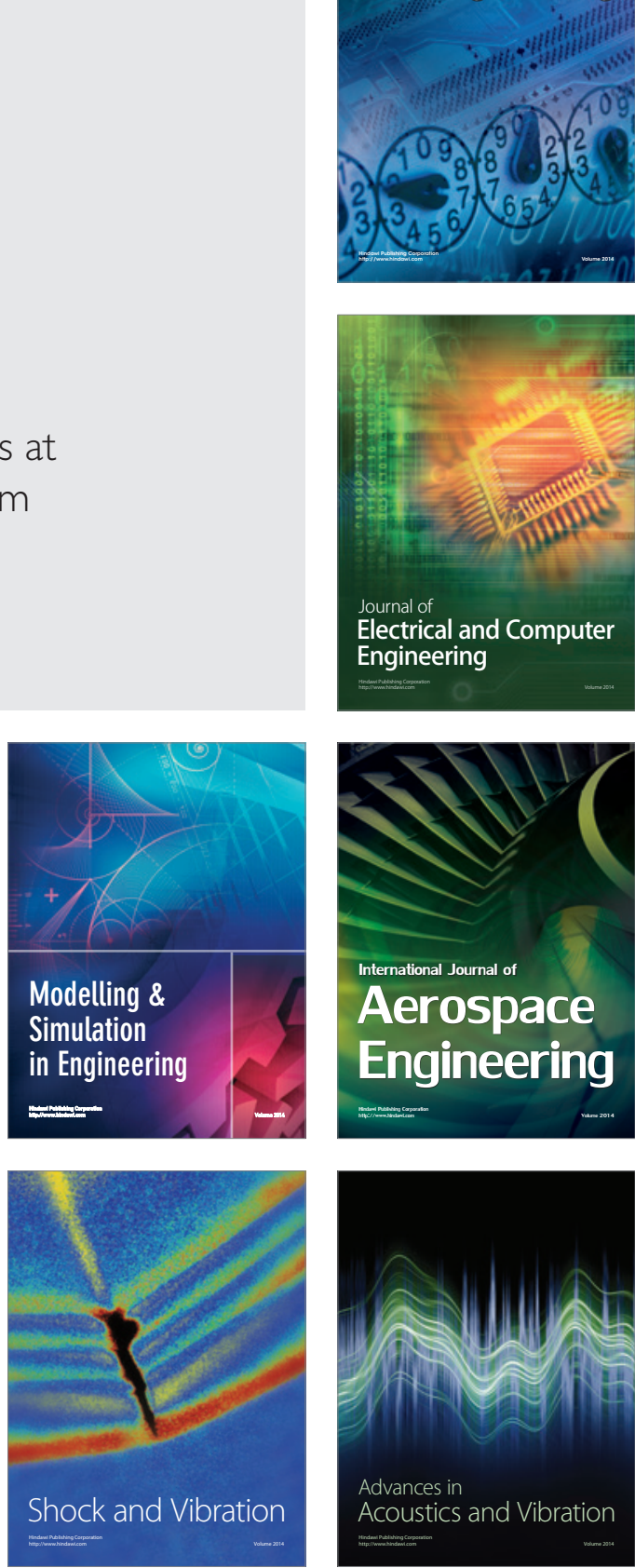